\title{
Targeted nano-sized drug design in cancer treatments: development of new nano-sized formulations for the use of anti-cancerogenic lisinopril in the treatment of colorectal cancer
}

\author{
Emre Avci ${ }^{1 *}$, Aslı Kara², Gamze Cagatay ${ }^{3}$, Gulcin Alp Avci ${ }^{3}$
}

\begin{abstract}
${ }^{1}$ Department of Molecular Biology and Genetics, Biochemistry, Faculty of Science and Arts, ${ }^{2}$ Department of Medical Services and Techniquies, Sungurlu Vocational High School, ${ }^{3}$ Department of Molecular Biology and Genetics, Biotechnology, Hitit University, Corum, Turkey
\end{abstract}

Received: 03 October 2020

Revised: 15 October 2020

Accepted: 16 October 2020

*Correspondence:

Dr. Emre Avci,

Email: avci.emre@yahoo.com

Copyright: $@$ the author(s), publisher and licensee Medip Academy. This is an open-access article distributed under the terms of the Creative Commons Attribution Non-Commercial License, which permits unrestricted non-commercial use, distribution, and reproduction in any medium, provided the original work is properly cited.

\begin{abstract}
Background: Colorectal cancer emerges as a serious health problem all over the world and results in approximately 700,000 deaths every year. Therefore, studies are carried out to develop alternative treatment methods to reduce the side effects of anticancer drugs in cancer treatment. Targeted nanoparticle therapies are tried to be developed especially with controlled drug release. Lisinopril, an angiotensin-converting enzyme inhibitor, is a widely used drug in the treatment of hypertension and has been shown to have anticancer activity on various types of cancer.

Methods: This study, blank and 3 different amounts of lisinopril loaded nanoparticles were prepared by triblock poly (lactic acid)-poly (ethylene glycol)-poly (lactic acid) (PLA-PEG-PLA) block copolymer which is a biocompatible and biodegradable polymer by using water/oil/water emulsion method and then characterized. The viability of the blank formulations in murine fibroblast (L929) cells, which is the healthy cell line, was determined within the scope of biocompatibility studies.

Results: The anticarcinogenic activity of lisinopril drug, blank and lisinopril loaded nanoparticles was determined in caco- 2 cells which are human epithelial colorectal adenocarcinoma cell line.

Conclusions: Lisinopril loaded nanoparticles were successfully prepared in this study. It is thought that increasing the amount of drug-loaded can be a promising approach to an alternative treatment method for the use of antihypertensive drugs in the treatment of colorectal cancer.
\end{abstract}

Keywords: Colorectal cancer, Antihypertensive drugs, Polymeric nanoparticle, Targeted drug design, Lisinopril

\section{INTRODUCTION}

Colorectal cancer is the third leading cause of cancerrelated deaths in men and the second leading cause of cancer-related deaths in women, resulting in approximately 700,000 deaths each year. ${ }^{1,2}$ Different methods are used to treat colorectal cancer. Although, surgery is the first or second treatment method in colorectal cancer treatment, radiotherapy and chemotherapy are more commonly applied methods for treatment. However, it is a big trouble that the resistance to chemotherapeutics develops after chemotherapeutic drug administration. ${ }^{3,4}$ However, it is a big trouble that the resistance to chemotherapeutics develops after chemotherapeutic drug administration. ${ }^{3}$ Whereas side effects such as high toxicity caused by traditional cancer drugs also destroy healthy cells. Therefore, in recent years some drugs which have known to target the specific cancer cells and have already approved for the treatment of diseases other than cancer are currently used for alternative 
drug development strategies. There are some studies in the literature showing that various drugs act as a preventive role for various cancer treatments. ${ }^{5}$

The fact that the pharmacokinetic properties and toxicity profiles of well-known Food and Drug Administration (FDA)-approved drugs used in the treatment of cancer today accelerate the passage to phase II and phase III studies and they provide a great advantage for their use in cancer treatment. In this context, it is considered a popular approach to examine the anticancer properties of several antihypertensive drugs. ${ }^{6}$ Lisinopril, one of the antihypertensive drugs, is an orally active long-acting angiotensin-converting enzyme (ACE) inhibitor drug used in the treatment of hypertension, heart failure, and acute myocardial infarction. ${ }^{7}$ Thus, ACE inhibition leads to a decrease in the amount of plasma angiotensin II, leading to a decrease in vasopressor effect and aldosterone secretion. It is known that angiotensin II, which is known to have an inhibitory effect on lisinopril, stimulates cancer development by affecting the apoptosis and proliferation of cancer cells and triggers the formation of cancer inflammatory microenvironment with angiogenesis. ${ }^{8}$ There are studies showing the anticancer properties of lisinopril, which is included in the ACE inhibitor class. ${ }^{6}$ However, it is thought that new generation delivery systems will provide a more effective and reliable treatment option in order to achieve therapeutic doses with a lower dose of this drug and to prevent its side effects. In the literature, the anticancer effect of free lisinopril has been demonstrated by in vivo and in vitro studies, but no treatment with the use of a carrier system has been encountered.

In recent years, polymeric nanoparticles are one of the most studied topics in combined therapies against many diseases. ${ }^{9}$ These nanoparticles have a high potential to be particularly effective in cancer therapeutic treatmen. ${ }^{10,11}$ In addition to the passive drug targeting advantage, nanoparticle formulations also offer the opportunity to increase the stability of drugs.

In this context, firstly, we aimed to load lisinopril, an antihypertensive drug, into nanoparticles prepared with triblock poly (lactic acid)-poly (ethylene glycol)-poly (lactic acid) (PLA-PEG-PLA), a biocompatible and biodegradable polymer, and then evaluate the anticancer activity of lisinopril loaded into nanoparticles in caco-2 cells.

\section{METHODS}

\section{Preparation of blank and lisinopril loaded nanoparticles with PLA-PEG-PLA block copolymer}

In this part of the study, Danafar et al method was used. ${ }^{12}$ Pharmaceutical:polymer (w/w) ratio, it was prepared and evaluated in 1:10, 1:2, 1:1, and 2:1. In the preparation of the proportions determined in the study, the PLA-PEGPLA polymer ratio was kept constant in order to evaluate the effect of the amount of drug on encapsulation. The amounts of the drug-loaded phase were varied. For ratio $1: 10,1 \mathrm{mg}$ of lisinopril dehydrate was dissolved in $1 \mathrm{ml}$ of distilled water, for ratio $1: 2,5 \mathrm{mg}$ of lisinopril dehydrate was dissolved in $1 \mathrm{ml}$ of purified water, for ratio 1:1, 10 $\mathrm{mg}$ of lisinopril dehydrate was dissolved in $1 \mathrm{ml}$ of purified water, for ratio $2: 1,20 \mathrm{mg}$ of lisinopril dehydrate was dissolved in $1 \mathrm{ml}$ of purified water.

The organic phase was prepared by the same procedure for all proportions; $10 \mathrm{mg}$ of PLA-PEG-PLA block copolymer was dissolved in $400 \mu \mathrm{l}$ of chloroform. The aqueous phase containing lisinopril was prepared by the same procedure for all proportions. This aqueous phase containing lisinopril was added dropwise onto the organic phase and after vortexing rapidly, the mixture was sonicated for 1 minute in ice. After sonication, the mixture was added dropwise to $4 \mathrm{~mL}$ of aqueous phase containing $0.4 \%$ polyvinyl alcohol (PVA) to obtain a double emulsion. The prepared emulsion was sonicated on ice for 5 minutes and then the emulsion was stirred on a magnetic stirrer for 2 hours, leaving the organic solvent to evaporate. At the end of the mixing period, the particles were precipitated at 13500 rotations per minute (rpm) at $+4{ }^{\circ} \mathrm{C}$ to remove the PVA from the emulsion. Afterward, the pellet was dispersed in pure water and centrifuged at $13500 \mathrm{rpm}$ for 2 times washing.

\section{Characterization of blank and lisinopril loaded nanoparticles with PLA-PEG-PLA block copolymer}

Particles were dispersed in pure water after washing, and size characterizations of freshly prepared particles were quickly analyzed with the ZetaSizer nano ZSP instrument. Blank nanoparticles were also prepared with the same preparation method as drug-loaded nanoparticles, only without adding lisinopril to the aqueous phase, and size characterizations were analyzed with the ZetaSizer nano ZSP device.

According to analyzes the drug:polymer ratio (1:1) in the most efficient volumes and amounts selected was determined, and the stage of determining the drug loading efficiency was initiated. For a ratio of $1: 1$ in the original volume:40 $\mathrm{mg}$ of lisinopril dehydrate was weighed and dissolved in $8 \mathrm{ml}$ of distilled water to form the aqueous phase. For the organic phase, $40 \mathrm{mg}$ of PLA-PEG-PLA block copolymer was dissolved in $1600 \mu$ l of chloroform. The aqueous phase containing lisinopril was added dropwise onto the organic phase. After vortexing quickly, the mixture was sonicated for 1 minute in ice. After sonication, the mixture was added dropwise to $16 \mathrm{~mL}$ aqueous phase containing $0.4 \%$ PVA to obtain a double emulsion. The prepared emulsion was sonicated on ice for 5 minutes and the organic solvent was left to evaporate by stirring the emulsion in magnetic stirrer for 2 hours. After the organic solvent was evaporated, the particles were precipitated at $+4{ }^{\circ} \mathrm{C}$ at $13500 \mathrm{rpm}$ in order to remove the PVA from the emulsion. Afterward, the pellet was dispersed in pure water and centrifuged at $13500 \mathrm{rpm}$ for 
2 times washing. After washing, the particles were dispersed in distilled water to a final volume of $10 \mathrm{ml}$ and lyophilized for 48 hours. Lisinopril was characterized before the amount of drug loaded into the particle was determined. The weight of the tube containing the samples before and after lyophilization was weighed and the loss during lyophilization was calculated.

\section{Determination of drug loading efficiency}

Characterization of lisinopril dehydrate by high pressure liquid chromatography analysis

As a result of the literature review, lisinopril was prepared in potassium dihydrogen orthophosphate buffer solution. For this, potassium dihydrogen buffer, adjusted to $\mathrm{pH} 2$ with orthophosphoric acid, was mixed with 95:5:0.1, potassium dihydrogen orthophosphate buffer:isopropyl alcohol:triethylenamine and the high pressure liquid chromatography (HPLC) were used with ultraviolet (UV) detector 210 . The determination was performed at $\mathrm{nm} .{ }^{13}$

\section{In vitro cell culture studies}

In vitro cell culture studies, Andima et al methods were utilized. ${ }^{14}$

\section{Evaluation of toxicity/biocompatibility of nanoparticles in L929 cell line}

L929 murine fibroblast cells were used in our biocompatibility studies. L929 cells were grown in Dulbecco's modified eagle's medium DMEM medium containing $10 \%(\mathrm{v} / \mathrm{v})$ fetal bovine serum (FBS), $50 \mathrm{U} / \mathrm{ml}$ penicillin and $50 \mu \mathrm{g} / \mathrm{ml}$ streptomycin in $25 \mathrm{~cm}^{2}$ flasks at $37{ }^{\circ} \mathrm{C}$ in an incubator containing $5 \% \mathrm{CO}_{2}$. When the cells became confluent (when they covered $80 \%$ of the flask surface), they were removed from the flask surface with the help of $10 \mathrm{X}$ trypsin-ethylenediaminetetraacetic acid (EDTA) solution and suspended in fresh culture medium. Cells were counted in the TC20 automated cell counter (Bio-Rad Inc, Hercules, California) using trypan blue before the experiment and they were inoculated into 96well plates at 5000 cells per well. Empty nanoparticles prepared in culture medium after an overnight incubation were diluted and applied to the wells at different concentrations. Only cells with culture medium were used as the control group. After the application, the plates were kept in the incubator for 24 and 48 hours. At the end of the incubation period, $20 \mu 1$ of CellTiter96®AQueous solution (Promega, Biotech AB) was added to each well. After 4 hours of incubation, absorbance was measured in a microplate reader at a wavelength of $490 \mathrm{~nm}$. Results were calculated assuming the cell viability of the control group as $100 \%$.

Evaluation of anticancer activity of lisinopril loaded nanoparticles
Caco-2 cells were grown in DMEM containing $10 \%$ (v/v) FBS, $50 \mathrm{U} / \mathrm{ml}$ penicillin and $50 \mu \mathrm{g} / \mathrm{ml}$ streptomycin in 25 $\mathrm{cm}^{2}$ flasks an incubator containing $5 \% \mathrm{CO}_{2}$ at $37^{\circ} \mathrm{C}$ using. Cells were seeded at 5000 cells per well for the experiment, and after an overnight incubation, the formulations were applied to the wells. Lisinopril drug, blank and lisinopril loaded nanoparticle formulations (drug: polymer-1:1) were prepared in cell culture medium at 4 different dilution rates (between $1 \mathrm{ppm}-0.125 \mathrm{ppm}$ ) to be applied to the wells. Only cells with culture medium were used as the control group. After application, plates were kept in incubator for 24 and 48 hours. At the end of the incubation period, $20 \mu \mathrm{l}$ of CellTiter96®AQueous solution was added to each well. After 4 hours of incubation, absorbance was measured in a microplate reader at a wavelength of $490 \mathrm{~nm}$. Results were calculated by assuming the cell viability of the control group as $100 \%$.

\section{Statistical analysis}

The findings of the study were evaluated statistically using IBM statistical package for the social sciences (SPSS) statistics 22.0 (IBM Co., Armonk, NY, USA). All data were given as mean \pm standard deviation. Statistical significance level was defined as 0.05. Statistical differences between groups were evaluated by one-way analysis of variance (ANOVA), one of the parametric tests, was used.

\section{RESULTS}

\section{Characterization of blank and lisinopril loaded nanoparticles with PLA-PEG-PLA block copolymer}

The results of the particle size, zeta potential, and polydispersity index (PDI) values of nanoparticles prepared in 4 different drugs in the low volume:polymer ratio are given in Table 1 . According to the particle size results, it was decided to prepare the nanoparticles in the original volume by choosing the ratio determined to have the optimum particle size, zeta potential, and PDI value. As seen in the table, the drug: polymer ratio of 1:1 was chosen as the optimum formulation with low particle size and PDI value and it was decided to continue the studies with this particle.

Table 1: Particle size, zeta potential, and PDI of lisinopril loaded nanoparticles.

\begin{tabular}{|llll|}
\hline $\begin{array}{l}\text { Drug: } \\
\text { polymer } \\
\text { ratio }\end{array}$ & $\begin{array}{l}\mathrm{Z} \text {-average } \\
(\mathbf{n m})\end{array}$ & PDI & $\mathbf{m V}$ \\
\hline $\mathbf{1 : 1 0}$ & $285.7 \pm 87.6$ & $0.23 \pm 0.02$ & $0.22 \pm 0.01$ \\
\hline $\mathbf{1 : 2}$ & $324.9 \pm 72.6$ & $0.28 \pm 0.03$ & $-5.45 \pm 0.8$ \\
\hline $\mathbf{1 : 1}$ & $280.2 \pm 26.4$ & $0.24 \pm 0.02$ & $-17.3 \pm 0.02$ \\
\hline $\mathbf{2 : 1}$ & $283.9 \pm 32.1$ & $0.26 \pm 0.01$ & $-13.5 \pm 0.01$ \\
\hline
\end{tabular}


However, since it is thought that the size of the 1:2 formulations with a high particle size may be higher due to the higher drug loading capacity, the study was repeated with the formulations of both nanoparticles, $1: 1$ and $1: 2$ ratios.

Table 2: Particle size, zeta potential, and PDI of lisinopril loaded and empty nanoparticles (NP).

\begin{tabular}{|ll|ll|}
\hline $\begin{array}{l}\text { Drug:polymer } \\
\text { ratio }\end{array}$ & $\begin{array}{l}\text { Z-average } \\
(\mathbf{n m})\end{array}$ & PDI & \multicolumn{1}{c|}{$\mathbf{m V}$} \\
\hline 1:2 empty NP & $334.5 \pm 98.8$ & $0.24 \pm 0.03$ & - \\
\hline $\begin{array}{l}\text { 1:2 Lisinopril } \\
\text { loaded NP }\end{array}$ & $368.9 \pm 93.5$ & $0,28 \pm 0.02$ & - \\
\hline 1:1 empty NP & $203.8 \pm 26.8$ & $0.22 \pm 0.01$ & - \\
\hline $\begin{array}{l}\text { 1:1 Lisinopril } \\
\text { loaded NP }\end{array}$ & $365.7 \pm 47.3$ & $0.26 \pm 0.01$ & - \\
\hline & & & $13.5 \pm 2.02$ \\
\hline
\end{tabular}

In the next step of the study, empty and drug-loaded nanoparticles were prepared with 1:2 and 1:1 drug-polymer ratio, and their characterizations in terms of particle size, zeta potential, and PDI values were analyzed with zeta sizer. Analysis results are given in Table 1, and 2 (Figures $1-4)$.

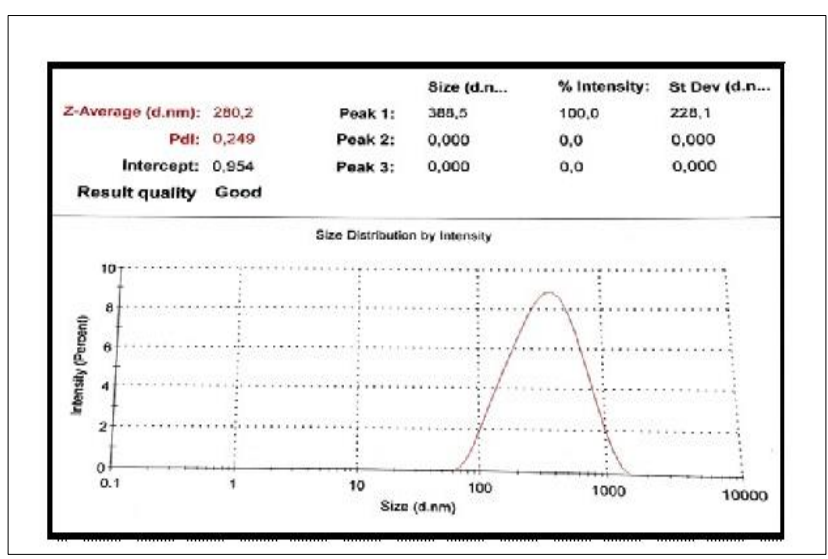

Figure 1: Size distribution chart of the drug to polymer ratio 1:1 formulation.

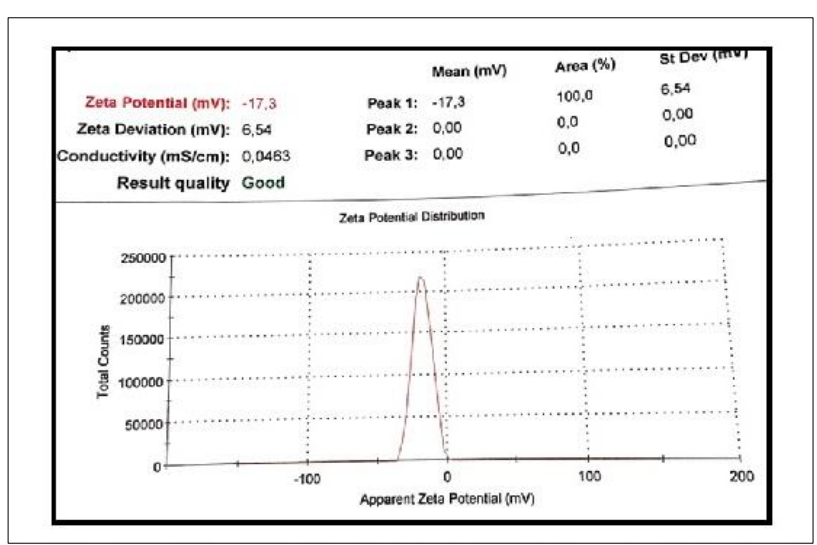

Figure 2: Zeta potential plot of the drug to polymer ratio 1:1 formulation.

The nanoparticles prepared in original volume were lyophilized for long-term storage. The weight of the tube containing samples before and after lyophilization was weighed and the loss during lyophilization was calculated.

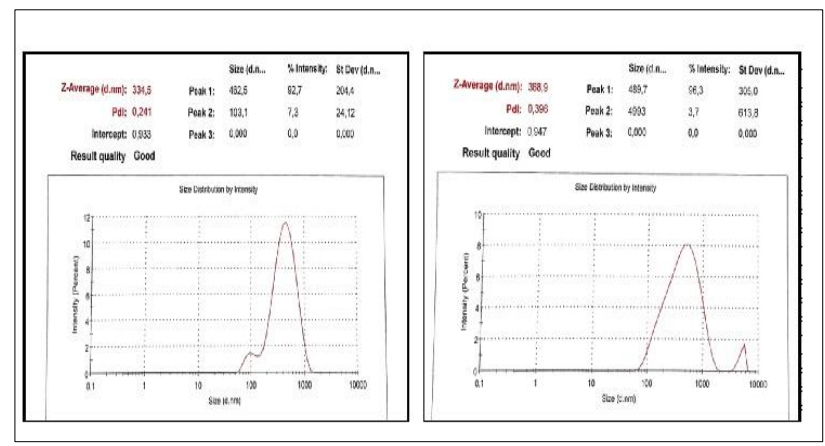

Figure 3: Size distribution graph of empty and lisinopril loaded nanoparticles prepared in 1:2 ratio.

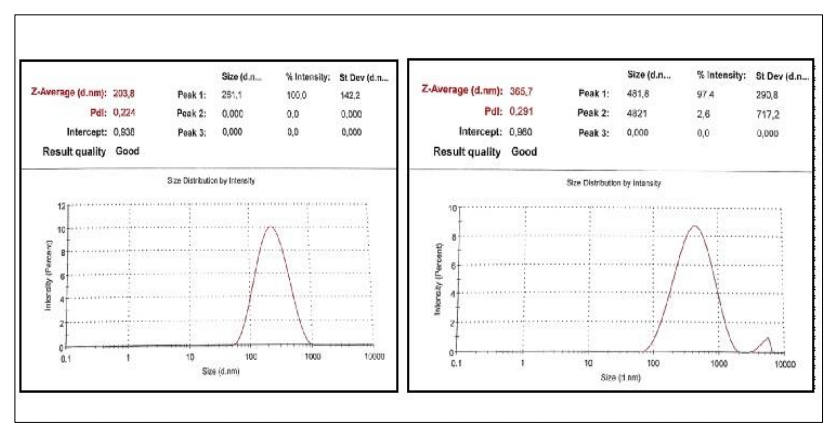

Figure 4: Size distribution chart of empty and lisinopril loaded nanoparticles prepared at a 1:1 ratio.

Table 3: Concentration versus field findings.

\begin{tabular}{|ll|}
\hline Concentration $(\mathrm{mg} / \mathrm{l}-\mathrm{ppm})$ & Field \\
\hline $\mathbf{1}$ & $0.7819 \pm 0.23$ \\
\hline $\mathbf{3}$ & $1.5088 \pm 0.48$ \\
\hline $\mathbf{4}$ & $2.1546 \pm 0.50$ \\
\hline $\mathbf{5}$ & $2.7942 \pm 0.61$ \\
\hline $\mathbf{6}$ & $3.3337 \pm 0.90$ \\
\hline $\mathbf{1 0}$ & $3.9551 \pm 0.96$ \\
\hline
\end{tabular}

Efficiency

$=$ Weight of tube (mg) after lyophilization

- tube weight $(\mathrm{mg}) \times 100$

Total amount of substance $(\mathrm{mg})$

$$
=5506.7-5462.3 \times 100 \div 80
$$

The efficiency obtained was determined as $55.5 \%$.

Lisinopril was characterized before the amount of drugloaded into the particle was determined. 


\section{Studies for determination of drug loading efficiency}

According to the literature data, it was tried to extract lisinopril from the particle by incubating it in an ultrasonic bath for 20 minutes in orthophosphate buffer as a different solvent. At the same time, concentrations of 1, 2, 3, 4, 5, 6 $\mathrm{mg} / \mathrm{l}$ of free lisinopril in buffer solution were prepared and given to HPLC. A plot of concentration versus area was created and the findings were given in Table 3 and Figure 5.

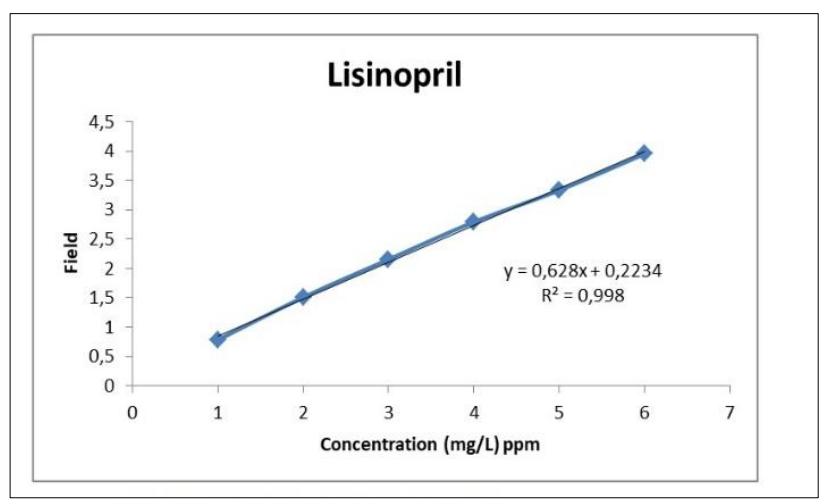

Figure 5: Calibration chart of lisinopril dehydrate.

Lisinopril dehydrate has been determined to be linear at the specified concentrations. Later, the lisinopril entrapped nanoparticle was extracted in a buffer solution at a rate of $1 \mathrm{mg} / \mathrm{ml}$ and then given to HPLC. According to the results of the study, the concentration of lisinopril imprisoned in the nanoparticle was determined as low as approximately 1 $\mathrm{mg} / \mathrm{l}(1 \mathrm{ppm})$ by HPLC.

\section{In vitro cell culture studies}

Evaluation of empty nanoparticles toxicity/ biocompatibility in L929 cell line

The graphs of the 24 and 48-hour biocompatibility in the L929 cell line of the empty nanoparticles prepared in 1:1 drug:polymer ratio selected. Comparison with control cells showed that empty nanoparticle formulations did not exhibit any cytotoxicity after 24 and 48 hours of application on healthy fibroblast cells. Thus, the biocompatibility of drug-free empty nanoparticles was confirmed.

\section{Evaluation of anticancer activity of lisinopril loaded nanoparticle}

As a result of the 24-hour experiment, it was determined that the applied empty and drug-loaded formulations showed similar results with the control group. Thus, it was observed that drug-loaded formulations did not have a cytotoxic effect on colorectal cancer cells within 24 hours. At the same time, it was determined that the drug did not have a cytotoxic effect at the end of 24 hours in caco-2 cells, where only lisinopril was administered at different concentrations.

According to the results of the 48-hour experiment, it was determined that lisinopril loaded nanoparticles prepared at a ratio of $1: 1$ cause a cytotoxic effect up to $16 \%$ at varying concentrations. This was attributed to the very low amount of drug-loaded. However, it has been shown that it has a certain anti-cancer effect when compared with the lisinopril drug administered at the same dilution rates.

\section{DISCUSSION}

It has a very important role in the future of targeted, personalized medicine with controlled drug release. Polymeric nanoparticles are one of the most studied topics for nanomedicine, especially in combined therapies against cancer. ${ }^{9}$ There is great interest in the potential of polymeric nanoparticles to be effective in therapeutic treatment in personalized cancer medicine. ${ }^{10,11}$ Particle size, morphology, polymeric material selection and processing techniques are important to determine the ideal polymeric nanostructures for more efficient and targeted delivery of drugs used in treatment. ${ }^{11}$ For this reason, a targeted drug design was considered in our study.

Antihypertensive drugs frequently used in the treatment of hypertension, and its anticancer activity has been determined on various types of cancer such as breast and liver. ${ }^{15}$ Sharkawi et al evaluated the cytotoxicity of some ACE inhibitor drugs by in vitro studies. Neutral red and trypan blue test methods were used against cell lines transformed with HeLa, hepatoma G2 (HepG2), MCF7, and Ehrlich ascities carcinoma cells (EACC) in the studies. All three drugs have been reported to show cytotoxicity against HeLa, HepG2, and MCF-7 cells. Lisinopril was shown to be the most potent cytotoxic drug against HepG2 cells with an inhibitory concentration (IC50) $=33.8 \pm 88.4$ $\mu \mathrm{g} / \mathrm{ml}$ at a concentration of $300 \mu \mathrm{g} / \mathrm{ml}$. As a result of the study, it was reported that, at high concentrations, the three drugs tested were lethal in vitro for endometrial, cervical, hepatic, and breast cancer cells. ${ }^{6}$ Babu et al reported that lisinopril has a significant inhibitory effect on carcinoma of cervical cells in a concentration-dependent manner. ${ }^{16}$ Considering the frequent use of these drugs, in our study we evaluated the effect of lisinopril, an antihypertensive drug active ingredient, as an alternative cancer treatment agent and the effectiveness of its form confined to the nanoparticle in the treatment.

Most of the current nano-applications report that the low drug loading associated with excess carrier material may have a disadvantage. Extensive use of carrier materials may cause systemic toxicity, especially in intravenous. ${ }^{17}$ Therefore, reducing the use of carrier materials to ensure therapeutic effect and function is seen as a promising alternative approach to solving these problems. ${ }^{17,18}$ For this reason, in our study, a 1:1 ratio (drug:polymer) was chosen as the optimum formulation with low particle size and PDI value. In addition, the study was repeated with both 
nanoparticle formulation ratios (1:1 and 1:2) for higher particle size formulations (1:2), due to the higher drug loading capacity of the size and for comparison.

Viability tests in cells are frequently used to evaluate the safety of biologically applied materials. ${ }^{19,20}$ Especially, nanomaterials and drugs, which are being used frequently today, cause different biological effects. Toxicity tests are often performed to determine biological effects on cells. ${ }^{21}$ In vitro MTT testing is widely used to measure cytotoxicity tests, cellular metabolic activity, and proliferation. ${ }^{22}$ CellTiter96®AQueous, which has high sensitivity in recent years, was used in our study. According to our cytotoxicity test results, it was determined that lisinopril loaded nanoparticles prepared at a ratio of 1:1 caused a cytotoxic effect up to $16 \%$ in varying concentrations. This data was attributed to the low drug load.

However, it has been observed that lisinopril, which is applied increasingly, has an anti-cancer effect.

\section{CONCLUSION}

In conclusion, the confinement of the antihypertensive lisinopril in the nanoparticle and the targeted anticancer activity in the colorectal cancer line has not been studied before. For this reason, it is thought that our study can bring innovation to the literature. When the characterization findings of the prepared empty and drug-loaded nanoparticles were evaluated, it was seen that they were compatible with the literature. In vitro toxicity of lisinopril loaded nanoparticles on caco- 2 cells was determined. It is among our predictions that higher anticancer activity will be achieved as the number of arrested drug increases.

Funding: Hitit University Research Fund (BAP) under grant number FEF19001.18.001

Conflict of interest: None declared

Ethical approval: The study was approved by the Institutional Ethics Committee

\section{REFERENCES}

1. Torre LA, Bray F, Siegel RL, Ferlay J, Lortet-Tieulent J, et al. Global cancer statistics, 2012. CA Cancer J Clin. 2015;65(2):87-108.

2. Abhishek B, Melissa W, Samir G. Colorectal cancer is a leading cause of cancer incidence and mortality among adults younger than 50 years in the USA: a SEER-based analysis with comparison to other youngonset cancers. J Investig Med. 2017;65(2):311-5.

3. Mármol I, Sánchez-de-Diego C, Pradilla Dieste A, Cerrada E, Rodriguez Yoldi MJ. Colorectal Carcinoma: A General Overview and Future Perspectives in Colorectal Cancer. Inter J Mol Sci. 2017;18(1):197.

4. Behzad M, Ali M, Sadaf D, Solmaz S, Behzad B. Adv Pharm Bull. The Different Mechanisms of Cancer Drug Resistance: A Brief Review. 2017;7(3):339-48.
5. Latha GS, Prasad SBC, Rao R. In vitro anti-cancer activities of few antihypertensive agents against carcinoma of scalp by MTT assay. J Chem Bio Phy Sci. 2011;1(2):299-303.

6. Sharkawi FZE, Shemy HAE, Khaled H. Anticancer activity of some commercial antihypertensive drugs by Neutral Red assay. Life Sci J. 2013;10(1):609-13.

7. Raia JJ, Barone JA, Byerly WG, Lacy CR. Angiotensin-converting enzyme inhibitors: a comparative review. DICP. 1990;24(5):506-25.

8. Munro MJ, Wickremesekera AC, Davis PF, Marsh R, Tan ST, et al. Renin-angiotensin system and cancer: A review. Integr Cancer Sci Therap. 2017;4.

9. Aniket G, Janel K, Brandon P, Prakash R. Targeting Cancer using Polymeric Nanoparticle mediated Combination Chemotherapy. Int $\mathrm{J}$ Nanomed Nanosurg. 2016;2(3):10.

10. Shin HC, Alani AW, Cho H, Bae Y, Kolesar JM,et al. A 3-in-1 polymeric micelle nanocontainer for poorly water-soluble drugs. Mol Pharm. 2011;8(4):1257-65.

11. Honary S, Zahir F. Effect of Zeta Potential on the Properties of Nano-Drug Delivery Systems - A Review (Part 2) Tropical J Pharm Res. 2013;12:26573.

12. Danafar H, Rostamizadeg, Davaran S, Hamidi M. PLA-PEG-PLA copolymer-based polymersomes as nanocarriers for delivery of hydrophilic and hydrophobic drugs: preparation and evaluation with atorvastatin and lisinopril. Drug Dev Ind Pharm. 2014;40(10):1411-20.

13. Chander V, Mohan M, Seth R, Singh P, Singh R, Gupta S. Development and Validation of RP-HPLC Method for the Estimation of Lisinopril in Tablet Dosage Form. Anal Chem Lett. 2012;2(5):309-13.

14. Andima M, Costabile G, Isert L, Ndakala AJ, Derese $S$, Merkel OM. Evaluation of $\beta$-Sitosterol Loaded PLGA and PEG-PLA Nanoparticles for Effective Treatment of Breast Cancer: Preparation, Physicochemical Characterization, and Antitumor Activity. Pharmaceutics. 2018;10(232):1-18.

15. Makar GA, Holmes JH, Yang YX. Angiotensinconverting enzyme inhibitor therapy and colorectal cancer risk. J Natl Cancer Inst. 2014;106(2):374.

16. Babu AP, Swarna Latha G, Bhavani Charan Prasad S, Ramachandra Rao CSV. In Vitrocancer activities of few anti-hypertensive agents against carcinoma of cervix by MTT assay. J Pharm Res Rev. 2011;1:1-3.

17. Shen S, Wu Y, Liu Y, Wu D. High drug-loading nanomedicines: progress, current status, and prospects. Int J Nanomedicine. 2017;12:4085-109.

18. Cai K, He X, Song Z, Yin Q, Zhang Y, Uckun FM, Jiang $\mathrm{C}$, Cheng J. Dimeric drug polymeric nanoparticles with exceptionally high drug loading and quantitative loading efficiency. J Am Chem Soc. 2015;137(10):3458-61.

19. Grenha A, Grainger CI, Dailey LA, Seijo B, Martin GP, Remuñán-López C, Forbes B. Chitosan nanoparticles are compatible with respiratory epithelial cells in vitro. Eur $\mathbf{J}$ Pharm Sci. 2007;31(2):73-84. 
20. Derman S, Mustafaeva AZ, Canim AS, Mansuroglu B., Kizilbey K, et al. The Study Of Synthetic Peptide Loaded PLGA Nanoparticles Cytotoxicity In Vitro. Fresenius Environ Bull. 2017;26(2a):1646-53.

21. Vijayakumar S, Ganesan S. In vitro cytotoxicity assay on gold nanoparticles with different stabilizing agents. J Nano. 2012;14.

22. Abid NBS, Rouis Z, Nefzi F, Souelah N. Aouni M. Evaluation of dimethylthiazol diphenyl tetrazolium bromide and propidium iodide inclusion assays for the evaluation of cell viability by flow cytometry. J Appl Pharm Sci. 2012;2:10-4.

Cite this article as: Avci E, Kara A, Cagatay G, Avci GA. Targeted nano-sized drug design in cancer treatments: development of new nano-sized formulations for the use of anti-cancerogenic lisinopril in the treatment of colorectal cancer. Int J Basic Clin Pharmacol 2020;9:1629-35. 